\title{
Utilización de Azadirachta Índica para el desarrollo de competencias multidisciplinarias estudiantiles en investigación ${ }^{1}$
}

\section{Azadirachta Índicass utilization for the development of multidisciplinary student competitions in investigation}

DOI: http://dx.doi.org/10.17981/cultedusoc.9.3.2018.88

Artículo de investigación. Fecha de recepción: 15/06/2018. Fecha de aceptación: 27/11/2018

Fredys Barraza Rojano;

Damiris Caballero Ospino; Jaime Ospino; Ana Pacheco Díaz;

Yamile Mourad Escobar y Éricka Camargo Amador ${ }^{3}$

Institución Educativa Departamental Luis Carlos Galán Sarmiento (Colombia)

barrazafredyro@gmail.co

Para citar este artículo:

Barraza, F., Caballero, D., Ospino, J., Pacheco, A., Mourad, Y. y Camargo, É. (2018). Utilización de Azadirachta Índica para el desarrollo de competencias multidisciplinarias estudiantiles en investigación. Cultura. Educación y Sociedad 9(3), 749-754. DOI: http://dx.doi. org/10.17981/cultedusoc.9.3.2018.88

\section{Resumen}

El presente artículo tiene como objetivo fortalecer las competencias en investigación de los estudiantes de la I.E.D Agroambiental Luis Carlos Galán Sarmiento utilizando la Azadiracha Indica o árbol de Neem como un recurso pedagógico desde una mirada multidisciplinar, metodológicamente se implementó la técnica de la observación participante y como instrumento el registro en diario de campo, y la población participante estuvo conformada por 50 estudiantes. Durante la ejecución del proyecto se integraron componentes curriculares de las Ciencias Naturales, Ciencias Sociales, Lengua Castellana y Matemáticas ofreciendo un abordaje interdisciplinario del objeto de investigación y generando aprendizajes significativos, donde los estudiantes adquirieron y fortalecieron destrezas en investigación.

Palabras clave: Azadirachta Índica o árbol de Neem, educación ambiental, estrategia pedagógica.

\section{Abstract}

The present article has as aim strengthen the competitions in investigation of the students of the I.E.D Environmental Agro Luis Carlos Galán Sarmiento using the Azadiracha Indíca or Neem's tree as a pedagogic resource from a multidisciplinary look, methodologically participant implemented the technology of the observation and since I orchestrate the record in field diary, and the population participant was shaped by 50 students. During the execution of the project components joined curriculum of the Natural Sciences, Social Sciences, Castilian Language and Mathematics offering an interdisciplinary boarding of the object of investigation and generating significant learnings, where the students acquired and strengthened skills in investigation.

Keywords: Azadirachta Indica or Neem's tree, environmental education, pedagogical strategy.

1 Este artículo ha sido derivado del programa de fortalecimiento de la cultura ciudadana y democrática CT+I a través de la IEP apoya en TIC en el Departamento de Magdalena: CICLON

2 Líder del grupo de investigación "Giviro".

3 Docentes de la institución Educativa Departamental Luis Carlos Galán Sarmiento y miembros del Grupo de investigación "Giviro".

- The author; licensee Universidad de la Costa - CUC. 


\section{Introducción}

LaAzadirachtaÍndica (árbol de Neem), es un árbol considerado como multipropósito gracias a su resistencia a la sequía, su capacidad para desarrollarse en cualquier tipo de suelo y soportar altas temperaturas; por sus propiedades químicas, es muy apreciado por la industria de la medicina; para el manejo de plagas y como materia prima de carbón y madera (EcuRed, 2016). Este árbol, que crece de forma silvestre en el municipio de Plato (Magdalena) no es reconocido, ni aprovechado en todo su potencial, razón por la cual se desarrolló esta investigación utilizándose como recurso pedagógico para fortalecer las competencias en investigación de los estudiantes a través de la construcción de proyectos de investigación en el aula escolar.

En este sentido el uso de plantas como recurso pedagógico para el proceso de enseñanza permite que el estudiante explore en sus conocimientos previos y nuevos llegando a modificar la practica pedagógica del maestro debido a que la dinámica de aprendizaje se transforma, promoviendo también el espíritu científico y pensamiento crítico en los estudiantes puesto que incentiva a buscar las explicaciones y relaciones entre los recursos usados y el proceso de investigación (Cuellar, 2007 citado en Martinez, 2015). Además este tipo de estrategias apuntan a que no solo aprendan y se involucren los investigadores y la población participante sino; que también estén presentes los demás actores de la comunidad educativa, asimismo se da apertura a que se haga un reconocimiento a su entorno y a los recursos que se encuentran en este, valorando su cultura y originando en general un sentido de pertenencia y motivación para mantener estas herramientas investigativas y ver en ellas un beneficio posterior en relación a los resultados que se obtengan (Martinez Quiñones, 2015).

La investigación como un proceso pedagógico cobra un valor significativo en la medida de que implementa el dialogo de saberes y este es visto como un elemento relevante ya que permite, que los participantes reflexionen acerca de la problemática de estudio y cuál es el impacto de los resultados obtenidos, y además que puedan identificar cual es la pertinencia de los resultados en función a las soluciones que se necesitan en el entorno (Beltran, Silva, Linares y Cardona, 2010). En este orden este tipo de proyectos formativos desde el aula provocan una potencialización del desarrollo comunitario derivando de este posibilidades de multiplicación de ideas y conocimientos que afiancen las habilidades y competencias en investigación de los estudiantes y la transmisión de saberes (Beltran, Silva, Linares y Cardona, 2010).

En cuanto a cómo los proyectos de investigación contribuyen al sistema educativo se plantea que estos asienten en la planificación de los planes curriculares colocándose en práctica en los salones de clases dando paso a que se dé una reforma en cómo se implementan las estrategias de enseñanza-aprendizaje, de este mismo modo se abonan otros aspectos como la innovación en cuanto que las clases cambian su metodología tradicional, ya que el docente busca otros espacios diferentes al aula y contempla el desarrollo multidisciplinar desde el objetivo de investigación (Choque, 2004). Por otra parte de este tipo de estrategias subyacen temáticas importantes a tratar como lo son las soluciones al medio ambiente y el emprendimiento que por medio de la experiencia que se tenga en la investigación se pueden aportar al mantenimiento y preservación ambiental; en cuanto al 
uso que se le dé a las plantas implementadas, y dependiendo de las propiedades de estas se pueden satisfacer otras necesidades como enfermedades, infectas de animales y cultivos, entre otros aspectos con la creación de productos a base orgánica (Rodriguez, 2010).

Por lo anterior los maestros investigadores implementaron el uso del árbol de Neem como un recurso pedagógico que ayude a los estudiantes a comprender y apropiarse del proceso de investigación, y que posteriormente estos a través de las reflexiones y comprensión de los resultados puedan proponer soluciones a distintas problemáticas tanto en la institución educativa, como en la comunidad en general del municipio teniendo un rol activo en el proceso de aprendizaje ya que ellos serán los artífices principales de la experiencia, y multiplicadores de conocimiento generando una motivación para fomentar el pensamiento analítico, crítico y científico.

\section{Metodología}

La metodología de estudio puesta en práctica se desarrolla desde un enfoque cualitativo con un diseño descriptivo debido a que se detallarán las características de cada proceso de crecimiento del árbol (Hernández, Fernández y Baptista, 2014); entonces se la Azadiracha Indica o árbol de Neem como recurso pedagógico para fortalecer competencias y destrezas en investigación de los estudiantes.

\section{Participantes}

La población participante del estudio corresponde a cincuenta (50) estudiantes de la I.E.D Luis Carlos Galán Sarmiento de la sede Villa Rosa de los grados de básica primaria, con edades entre 6 a 12 años.

\section{Técnicas e instrumentos}

En la investigación debido al enfoque y diseño se seleccionó como técnica de recolección de información la entrevista, la cual es definida como una conversación o dialogo entre dos o varias personas en donde se realizan preguntas y se escuchan respuestas (Vargas, 2012). Como instrumento de información se implementa el diario de campo en el que se consignan todas las situaciones y desarrollo de actividades que se impartan en el proceso de investigación y las acciones y comportamientos de los actores (Hernández, Fernández, y Baptista, 2014).

\section{Procedimiento}

Las actividades que se llevaran a cabo para la ejecución del proyecto se organizaron de la siguiente manera, inicialmente se organizó a los estudiantes por grupos teniendo en cuenta el grado y se les asigna realizar una consulta acerca del tema en estudio, posteriormente se elaboraron talleres en el que se les enseño a los estudiantes las particularidades de la planta, conceptos matemáticos para la siembra, uso de creencias sobre el árbol para la creación de cuentos y remisión a fuentes de información informales como entrevistas a los ancianos del pueblo.

\section{Resultados}

A continuación en la tabla 1 se describen los resultados obtenidos de la implementación de las actividades realizadas en el proyecto de investigación, entre estas se encuentran jornadas de consulta y talleres con los estudiantes. 
En la presente actividad los estudiantes consultaron inicialmente sobre que era el árbol de Neem y sus características, en qué país se cultiva de manera original y en que otros lugares del mundo, y cuáles son los beneficios de la planta. Durante este taller los estudiantes en cada computador investigaron la información

Actividad 1.

Reconocimiento:

En la presente actividad los estudiantes con el acompañamiento de docentes del área de ciencias sociales desarrollaron una consulta en una sala de cómputo de la institución en la que consultaron sobre los orígenes del árbol de Neem.

\section{Actividad 2.}

Taller de capacitación:

En esta actividad los estudiantes expusieron los conceptos encontrados en la consulta anterior y reflexionaron acerca de los beneficios del árbol de Neem asesorados por un docente de ciencias naturales.

\section{Actividad 3.}

Taller de capacitación: En la siguiente actividad los estudiantes por instrucciones de un docente de matemáticas recibieron información acerca de las proporciones que se deben implementar en la siembra con relación a cantidad de terreno y semillas.

\section{Actividad 4.}

Taller de capacitación:

En este taller los estudiantes con el acompañamiento de un maestro de Lengua Castellana ya después de haber consultado sobre el proceso de siembra, cosecha y post cosecha incorporaron la creación de cuentos y revisión de mitos y leyendas que evoca el origen de la planta en la región. solicitada encontrando y socializando el contenido mostrándose interesados por conocer de un tema nunca antes tratado por ellos, y motivados por que estaban utilizando elementos tecnológicos para aprender y que eran diferentes a su clase normal.

Entre los resultados encontrados los estudiantes expresaron que el árbol de Neem tiene propiedades químicas que tienen componentes de los que se derivan insecticidas, ayudan al mejoramiento de enfermedades como la tuberculosis, propiedades antibacteriales, y ácidos como la quercetina y el ascórbico que son utilizados en frecuentemente en medicamentos. Además que se cultiva en la India como país pionero y que puede alcanzar una altura de 20 metros, y sus hojas, tallo, flores y frutos son beneficiosos para satisfacer necesidades de los seres humanos, ya que puede ser comestible, usarse para productos de aseo, medicamentos y fertilizantes.

Los estudiantes en este espacio por grupos respectivamente de cada grado expusieron las propiedades del árbol de manera inicial; es decir, como se siembra, como es su desarrollo y como se da la cosecha del fruto, y que productos surgen en la post cosecha y cuáles son los beneficios. En esta parte los estudiantes se documentaron al punto que se les noto de manera amplia la propiedad del tema exponiendo, estos diseñaron unas carteleras en las que graficaron los procesos y manifestaron que "profe, la actividad me gusta mucho, porque hemos hecho cosas diferentes además aprendo sobre cosas diferentes", "seño me gusta venir a los talleres porque estamos dándole provecho a un tema que casi nadie ha estudiado y utilizando un árbol que es de nuestro pueblo".

En el desarrollo de la presente actividad los estudiantes estuvieron más atentos de lo acostumbrados ya que manifestaban que cuando se da matemáticas todo es más complicado, por lo anterior el docente realizo el taller a partir de dinámicas y juegos en la que le enseño los conceptos numéricos de cómo debían sembrar los que corresponden a 20 bolsas por lotes de terreno y 5 semillas del árbol por cada bolsa y al mismo tiempo se registraron las medidas en centímetros de cuanto crecía la planta y el tiempo, reforzando temáticas de estudio como unidades de medida y números pares e impares.

En esta actividad los estudiantes se reunieron con una pareja de adultos mayores quienes les contaron leyendas de la región relacionadas con el árbol de Neem, entre las que se destacó la leyenda del hombre de Plato, Magdalena que cortaba las cabezas y las colgaba en los arboles de Neem para exhibirlas. Después de esto los estudiantes construyeron sus propias historias desde lo investigado de la planta añadiéndole un componente fantasioso e ilustrando los personajes de la historia, mostrándose muy interesados y creativos en la actividad.

Fuente: elaboración propia. 
Considerando los resultados obtenidos se contempla que los estudiantes participantes desarrollaron competencias en distintas áreas del saber cómo; Ciencias Naturales, Matemáticas y Lengua Castellana, ya que en el estudio se implementaron actividades desde diferentes disciplinas con el objetivo de hacer transversal el conocimiento y fortaleciendo competencias y habilidades en los estudiantes a partir de la investigación con elementos propios de su entorno. En este sentido se abona que contribuye el proyecto al embellecimiento de la institución por el hecho de la siembra y de manera indirecta la creación de conciencia ambiental y fomento de valores proambientales.

\section{Discusión}

Con la implementación de un proyecto de investigación enfocado al fortalecimiento de las competencias y habilidades en investigación las estrategias pedagógicas implementadas se consideran una forma idónea de enseñanza, debido a que los estudiantes aprehenden de manera efectiva y significativa, teniendo en cuenta que se hace uso de herramientas interactivas que contribuyen también a su desarrollo cognitivo.

En este mismo sentido surge que los estudiantes no solo robustecen sus destrezas desde una sola área del saber, sino; que por ser multidisciplinar integran distintos conocimientos que le permiten construir nuevos saberes e innovar en la forma de enseñanza de la institución, ya que desde la practica investigativa se evidencia que los estudiantes se sienten más interesados y pueden proponer nuevas ideas a partir de los vacíos que se identificaron en el proceso.

Finalmente derivado del estudio se plantea que los estudiantes en acompañamiento de los docentes, pueden desarrollar un plan de emprendimiento en el que se diseñen y se distribuyan productos medicinales o de fertilizantes, con el fin de que estos cuenten con un ingreso adicional y mejoren también su calidad de vida. Por otra parte también se puede elaborar un proyecto donde se trabaje de manera directa la educación ambiental y comportamiento proambiental con el objetivo de fomentar cultura en la comunidad estudiantil y administrativa.

\section{Referencias}

Álvarez, R. (1998). Pedagogía y didáctica. Pedagogía, 99.

Alvarado, L. y García, M. (2008). Características más relevantes del paradigma socio-crítico: su aplicación en investigaciones de educación ambiental y de enseñanza de las ciencias realizadas en el Doctorado de Educación del Instituto Pedagógico de Caracas. Sapiens: Revista Universitaria de Investigación, 9(2), 187-202.

Beltran, A., Silva, N., Linares, E. y Cardona, F. (2010). La etnobotánica y la educación geográfica en la comunidad rural Guacamayas, Boyacá, Colombia. Unipluri/versidad, 10(3). 1-11.

Calvo, S. y Corraliza, J. A. (1994). Educación ambiental: conceptos y propuestas. Madrid: CCS.

Choque, L. (2004). El Uso de Plantas Medicinales: Primeras Experiencias de Diversificación Curricular en el Distrito Educativo Charazanicurva. [Tesis].Universidad Mayor de San Simon, Cochabamba.

DANE. (2006). Informe de Censo Poblacional 2005. Recuperado de https://www. dane.gov.co/files/censo2005/PERFIL_ PDF_CG2005/47555T7T000.PDF

FAO. (2017). Estado de los bosques del mundo 2016. Recuperado de http://www. f a o o r g / n e w s r o o m / e s / fo cus/2006/1000247/index.html

Sunkel G. (2005). Las Tecnologías de la información y comunicación (TIC) en la educación de América Latina. Serie Políticas Sociales, 126. 1-170. 
Fuentes, H. (2009). Pedagogía y didáctica de la Educación Superior en la concepción de la universidad humana cultural una propuesta desde la Universidad Estatal de Bolívar. Guaranda: Universidad Estatal de Bolívar.

García, J. y Nando, J. (2000). Estrategias didácticas en educación ambiental. Malaga: Aljibe

Hervás, R. (2003). Estilos de enseñanza y aprendizaje en escenarios educativos. Granada: Grupo Editorial Universitario.

Leal, A. Z. (2005). Didáctica, pedagogía y saber. Bogotá, D.C.: Cooperativa Editorial Magisterio.

Lucio, R. (1989). Educación y pedagogía, enseñanza y didáctica: diferencias y relaciones. Revista Universidad de la Salle, (17), 35- 46.

Leal, A. Z. (2002). Los hilos de la palabra: pedagogía y didáctica. Bogotá, D.C.: Cooperativa Editorial Magisterio.

Lucini, F. (1996). Temas transversales y educación en valores. Madrid: Grupo Anaya.

Martinez, J. (2015). Diseño de un proyecto de aula para fortalecer el conocimiento, sobre el uso y aprovechamiento de las plantas medicinales en grado séptimo de la institución educativa niño jesús de pragra del bajo calima, distrito de buenaventura valle del cauca. [Trabajo de grado]. Universidad del Valle, Cali.

Mora, J. G. (2004). La necesidad del cambio educativo para la sociedad del conocimiento. Revista Iberoamericana de educación, 35(2), 13-37.
Ordoñez, C. y Castaño, C. (2011). Curso de Pedagogía y didáctica. Quito: Centro gráfico Ministerio de EducaciónDinse.

República de Cuba. (05 de septiembre de 2016). Proyecto de Constitucion de la republica de Cuba. Recuperado de http://www.parlamentocubano.cu/wpcontent/uploads/Tabloide-Constitución.pdf

Rodriguez, J. (2010). Uso y manejo tradicional de plantas medicinales y mágicas en el valle de sibundoy, alto putumayo, y su relación con procesos locales de construcción ambiental. $R e$ vista de la Academia Colombiana de Ciencias Exactas, Físicas y Naturales, 34(132). 309-326.

Unesco. (2004). Las Tecnologías de la Información y la Comunicación en la formación Docente. Recuperado de h t t p : // unesdoc.unesco.org/ images/0012/001295/129533s.pdf

Valverde, F. (1998). Plantas Útiles del Litoral Ecuatoriano. Guayaquil: Ministerio de Medio Ambiente -ECORAE- EcoCiencia. Herbario QCA-PUCE, Pontificia Universidad Católica del Ecuador.

Vasco, C. (1990). Algunas reflexiones sobre la pedagogía y la didáctica. Pedagogía, discurso y poder, 107-122.

Zambrano, A. (2016). Pedagogía y didáctica: esbozo de las diferencias, tensiones y relaciones de dos campos. Praxis \& saber, 7(13), 45-61. 